\title{
The properties of the circumnuclear regions in the Circinus galaxy
}

\author{
F. Massaro ${ }^{1,2}$, S. Bianchi ${ }^{1,3}$, G. Matt ${ }^{1}$, E. D'Onofrio ${ }^{1}$, and F. Nicastro ${ }^{4}$ \\ 1 Dipartimento di Fisica, Università degli studi Roma Tre, via della Vasca Navale 84, 0046 Roma, Italy \\ e-mail: massaro@roma2.infn.it \\ 2 Dipartimento di Fisica, Università degli studi di Tor Vergata, via della Ricerca scientifica 1, 00133 Roma, Italy \\ 3 XMM-Newton Science Operations Center, European Space Astronomy Center, ESA, Apartado 50727, 28080 Madrid, Spain \\ ${ }^{4}$ Harvard-Smithsonian Center for Astrophysics, 60 Garden Street, Cambridge, MA 02138
}

Received 24 December 2005 / Accepted 23 March 2006

ABSTRACT

\begin{abstract}
We analysed the spectra from the XMM-Newton and the three longest Chandra observations of the Circinus Galaxy, the X-ray brightest, Compton-thick Seyfert 2 galaxy, to derive the physical and morphological parameters of the circumnuclear matter. We compare the line and continuum parameters with extensive calculations performed with the well-known photoionisation code CLOUDY. The line spectrum cannot be explained by a single zone, as three different reflecting regions are required. The first has low ionisation, accounting for the prominent iron $\mathrm{K} \alpha$ line, and is likely to be identified with the "torus" envisaged in unification models for Seyfert galaxies; a lower limit of $0.05 \mathrm{pc}$ to the distance of this matter from the nucleus has been estimated, using new constraints on the ionisation state of iron $(\leq \mathrm{FeX})$. The second zone is moderately ionised and optically thin $(\tau \simeq 0.2)$, and it accounts for the oxygen to silicon lines. Finally, a third, highly ionised and optically thin reflector is required for the detection of the He-like iron line.
\end{abstract}

Key words. galaxies: nuclei - galaxies: Seyfert - X-rays: individuals: Circinus Galaxy

\section{Introduction}

In type 2 Seyfert galaxies, the obscuration of the nuclear X-ray emission allows us to observe and study spectral components produced by circumnuclear regions that in type 1 sources are outshined by the nuclear radiation. Particularly interesting are the results that can be obtained by studying the reflection of nuclear X-rays from the circumnuclear matter. Both neutral and ionised reflecting regions in Seyfert 2 galaxies give us indirect, scattered views of the interior of the central engine by Comptonscattering the nuclear continuum and producing copious X-ray line emission (e.g. Krolik \& Kallman 1987; Krolik \& Kriss 1995; Matt et al. 1996). Reflection from neutral matter, customarily attributed to the dusty torus envisaged in Seyfert unification models (Antonucci 1993) is a common feature of Seyfert 2 (e.g. Matt et al. 2000). Extended emission from X-ray line emitting gas in the ionisation cones of Seyfert galaxies was also seen in Chandra observations of NGC 4151 (Ogle et al. 2000), NGC 1068 (Brinkmann et al. 2002), and in Mrk 3 (Bianchi et al. 2005a), as well as in many other Seyferts (Bianchi et al. 2006). In principle, the reflecting regions allow us to map the spatial distribution of the gas and dust surrounding the nuclear regions and to derive the physical and chemical properties of such matter. To this purpose, analysing the emission spectral lines is particularly important (e.g. Matt et al. 1996; Netzer 1996; Krolik 1999, and references therein).

It is customary to classify Seyfert 2 galaxies as Comptonthick (i.e. $N_{\mathrm{H}} \geq 10^{24} \mathrm{~cm}^{-2}$ ) or Compton-thin (i.e. $N_{\mathrm{H}} \leq$ $10^{24} \mathrm{~cm}^{-2}$ ) sources. In this paper, we discuss the X-ray spectral features of one of the brightest and most studied Seyfert 2, the Circinus Galaxy, observed with XMM-Newton and Chandra. This source belongs to the Compton-thick class and its nuclear emission is obscured up to about $10 \mathrm{keV}$. Previous X-ray observations have shown many spectral lines from several elements in different ionisation states. Our XMM-Newton observation, characterised by a high $\mathrm{S} / \mathrm{N}$ ratio, gives the possibility of detecting low-energy emission lines useful for deriving the properties of the circumnuclear matter. In addition we also considered both new and archive Chandra observations (the latter discussed in Sambruna et al. 2001a,b) that, when combined, provide a high-quality line spectrum. We then used the code CLOUDY (http://www.pa.uky.edu/-gary/cloudy, vers.94: Ferland 1997) to calculate photoionisation models, and the resulting ionisation structure is compared with the results of our data analysis. In particular, we found that there is no way to explain the observed line spectrum using a single reflector model, contrary to what found by Bianchi et al. (2001) on the basis of the lower quality (and therefore less constraining) ASCA and BeppoSAX data.

This paper is organised as follows. Section 2 summarises previous observations on the Circinus Galaxy in different bands of the electromagnetic spectrum, and Sect. 3 describes the observations analysed in this paper and the data reduction. Section 4 reports on the spectral analysis, while the comparison with the photoionisation models is discussed in Sect. 5; conclusions are summarised in Sect. 6.

\section{Observational properties of the Circinus Galaxy}

The Circinus Galaxy hosts one of the nearest active galactic nuclei (AGN). A distance of $4 \mathrm{Mpc}$ was estimated for it by Freeman et al. (1997). The intense coronal lines (Oliva et al. 1994; Marconi et al. 1996), together with the variable $\mathrm{H}_{2} \mathrm{O}$ maser emission, the traces of an accretion disk (Nakai et al. 1995; Greenhill et al. 1997), the broad $\mathrm{H} \alpha$ line in polarized light (Oliva et al. 1998), and the reflection-dominated 2-10 keV X-ray spectrum (Matt et al. 1996), support the Seyfert 2 classification. The 


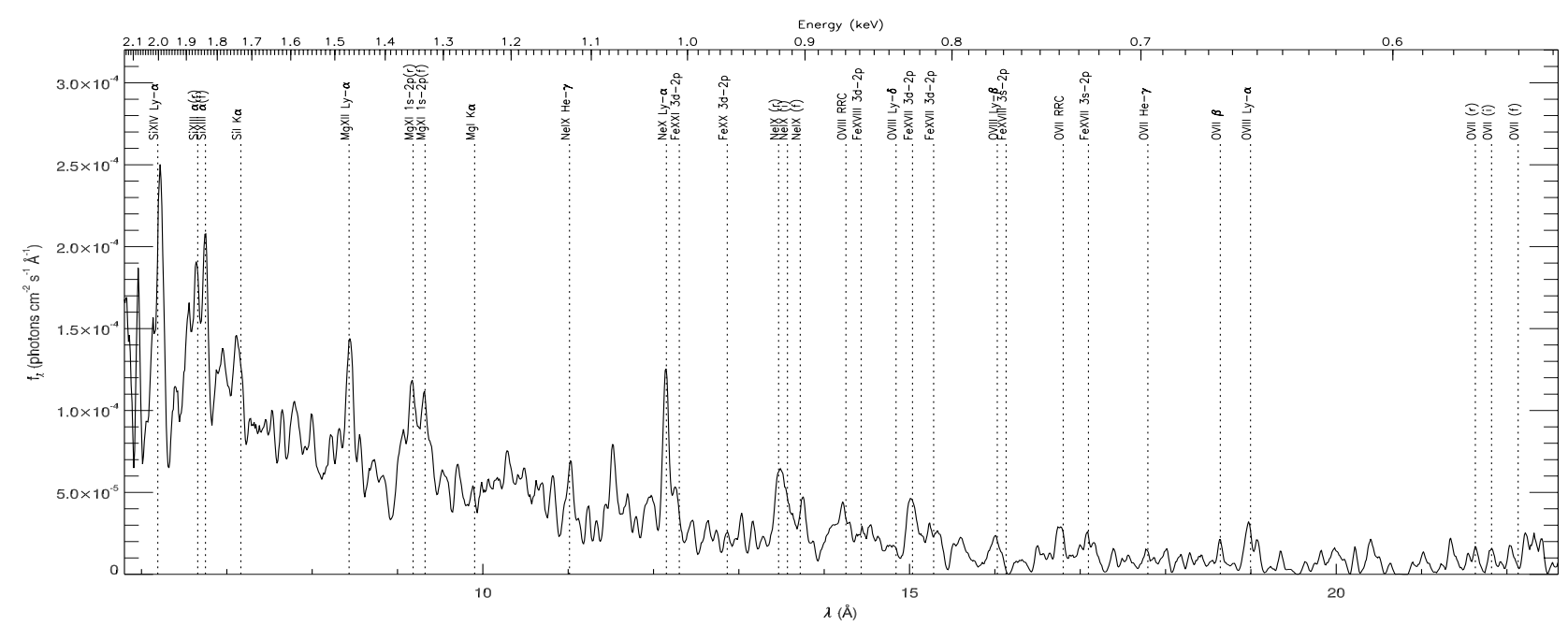

Fig. 1. The XMM-Newton RGS spectrum,with the main expected emission lines.

integrated IR luminosity is about $4 \times 10^{43} \mathrm{erg} \mathrm{s}^{-1}$, mostly due to the reprocessing of the intrinsic nuclear emission (Moorwood et al. 1996; Maiolino et al. 1998).

The Circinus AGN also shows an ionisation cone of [O III] (Marconi et al. 1994), in which linear optical filaments and compact knots were observed (Veilleux et al. 1997). The cone opening angle is about $90^{\circ}$, at a mean position angle (PA) of roughly $290^{\circ}$. Circinus also exhibits a kpc-scale nuclear outflow betrayed by bipolar radio lobes (Elmouttie et al. 1998), which are largely aligned to the minor axis of the galactic $\mathrm{H}$ I disk (Jones et al. 1999) and the ${ }^{12} \mathrm{CO}$ ring (Curran et al. 1998). A recent VLT observation (Prieto et al. 2004) has shown the presence of an [Si VII] ionisation bicone, whose size is compatible with a putative parsec-scale torus. Studies of the spectral energy distribution imposed constraints on the dust temperature of the torus around $300 \mathrm{~K}$ while hotter dust within a $1 \mathrm{pc}$ radius of the centre was not detected. This in turn supported the existence of highly obscuring material with column densities of $10^{24} \mathrm{~cm}^{-2}$, that must be located within $1 \mathrm{pc}$ of the core. The Circinus Galaxy was observed in X-rays for the first time during the ROSAT All Sky Survey (Brinkmann et al. 1994).

The ASCA observation (Matt et al. 1996) showed a flat X-ray spectrum with several emission lines, including a very prominent iron $\mathrm{K} \alpha$ line at about $6.4 \mathrm{keV}$. According to AGN unification models (Antonucci et al. 1993), these features were interpreted as the reflection of the intrinsic emission by circumnuclear matter (possibly the torus) surrounding the central engine. The absence of any spectral transmitted component up to $10 \mathrm{keV}$ in the ASCA band indicated an absorbing matter with a column density exceeding $10^{24} \mathrm{~cm}^{-2}$, making Circinus a Compton-thick source.

Circinus was observed above $10 \mathrm{keV}$ for the first time by BeppoSAX (Matt et al. 1999). At those energies, the intrinsic nuclear emission becomes visible and can be described well by a power law, with the photon index equal to 1.56 and an exponential cut off at $56 \mathrm{keV}$, absorbed by cold matter with $N_{\mathrm{H}} \simeq$ $4 \times 10^{24} \mathrm{~cm}^{-2}$ (Matt et al. 1999). On the other hand, the X-ray spectrum below $10 \mathrm{keV}$ is dominated by the cold reflection component (Matt et al. 1996; Guainazzi et al. 1999). The ASCA and BeppoSAX data were reanalysed later and compared with photoionisation models based on CLOUDY (Bianchi et al. 2001). The spectrum could be described as the reprocessing of nuclear emission by a single, mildly ionised reflector (but Sako et al. 2000, modelled it with two reflectors, one neutral one ionised). X-ray emission lines were also identified and studied by Sambruna et al. (2001b) based on Chandra data, who again model the line spectrum with two reflectors of different ionisation. Bianchi et al. (2002) reanalysed the Chandra spectrum and found evidence of the iron $\mathrm{K} \alpha$ "Compton shoulder", which was later confirmed by Molendi et al. (2003) who analysed the 5-13 keV XMM-Newton spectrum. The ratio between the Compton shoulder and the line core (Matt 2002) suggests identification of the reflector with the $N_{\mathrm{H}} \simeq 4 \times 10^{24} \mathrm{~cm}^{-2}$ absorber.

\section{Observations and data reduction}

\subsection{XMM-Newton}

The XMM-Newton data reduction has been described in Molendi et al. (2003), and we refer the reader to that paper for details. We recall here that the source was observed on 6-7 August 2001, for about $110 \mathrm{ks}$.

In Sect. 4 we will only discuss the EPIC/p-n spectrum. The spectrum was extracted from a 30 arcsec radius circle centred on the source peak. Two nearby sources lie within this radius, but were excluded by excising circles with 7 arcsec radius centred on the respective peaks (see Molendi et al. 2003, for a detailed discussion). The EPIC/MOS spectra are consistent with each other and with the p-n spectrum but, as they do not provide a very large increase in sensitivity, for the sake of simplicity we ignore them in the detailed spectral analysis. We did not analyse in detail the Reflection Grating Spectrometer (RGS; den Herder et al. 2001) because the field is pretty crowded, especially at low energies, and it would become difficult to disentangle the nuclear spectrum from those of the other sources. We reduced the RGS data using the standard SAS (version 6.1.0) meta-task rgsproc and the most updated calibration files available at the moment the reduction was performed (October 2005). The wavelength systematic uncertainty is $8 \mathrm{~m} \AA$ across the whole RGS sensitive bandpass. Since the RGS spectrum is approximately extracted from a rectangular region, centred on the target, $5.5^{\prime}$ high and all the pn field wide, it is contaminated by many nearby sources, in particular the two strong sources excluded from the EPIC pn region (see Molendi et al. 2003). This prevents us from performing a quantitative fit of the RGS spectra. Therefore, Fig. 1, which was generated by combining all the 1 st and 2 nd order spectra of the two RGS camera and smoothing the combined spectra through a convolution with a 5-spectral channel wide 
triangular function, is presented for illustrative purposes only, and only allows a qualitative analysis.

\subsection{Chandra}

The Circinus Galaxy was observed four times by the Chandra ACIS-S HETG (High Energy Transmission Gratings: Canizares et al. 2005). We consider here only the three longest observations, those performed on 6 June 2000, 2 June 2004 and 28 November 2004, for about $67 \mathrm{ks}, 56 \mathrm{ks}$, and $60 \mathrm{ks}$ of exposure time, respectively. The fourth observation was disregarded because it lasted only $8 \mathrm{ks}$. While the 2000 HETG spectra were already discussed in Sambruna et al. (2001b) and Bianchi et al. (2002), we present in this paper the other two observations for the first time. Data were reduced with CIAO 3.2.2 and CALDB 3.1.0. First-order MEG and HEG spectra were extracted following standard procedures. After having verified that the three spectra were consistent among themselves, we decided to combine them to enhance the $\mathrm{S} / \mathrm{N}$. This process was performed using the script ADD_GRATING_SPECTRA available on the CXC website. The final spectrum has a total exposure time of $174 \mathrm{ks}$.

\section{Spectral analysis}

Spectral analysis was been performed with the XSPEC software package. Errors refer to a $90 \%$ confidence level for one interesting parameter (i.e. $\Delta \chi^{2}=2.71$ ).

\subsection{XMM-Newton data analysis}

As in Bianchi et al. (2001), we fitted our data with a spectral continuum described as a combination of two models: a single power-law for soft X-ray emission and a Compton reflection model (pexrav model in XSPEC, Magdziarz et al. 1995), which describes the high energy part of the spectrum. We then added as many emission lines to this continuum model found from a direct inspection of residuals as are required. Our model is therefore:

$M=[P L+R C+$ emission lines $] \exp \left(-\sigma_{\mathrm{ph}} N_{\mathrm{H}}\right)$

where $\mathrm{RC}$ is a pure cold reflection component (pexrav model in XSPEC) with the power-law photon index of the illuminating continuum fixed to the value of $1.56\left(\Gamma_{0}\right)$ with a high energy cut off at $56 \mathrm{keV}\left(E_{\mathrm{c}}\right)$ as in Matt et al. (1999) and Bianchi et al. (2001). This continuum is produced by reprocessing of nuclear emission by circumnuclear Compton-thick matter. Then $N_{\mathrm{H}}$ is the cold absorption along the line of sight, due to our Galaxy and the Circinus Galaxy itself. In both absorption and reflection models, we assumed solar abundances for all the elements ( $\left.A=1, A_{\mathrm{Fe}}=1\right)$. The incident, absorbed continuum discovered by BeppoSAX is not present in our model because it is not directly observable in the XMM-Newton range, and PL is a power law model used to describe the soft X-ray continuum. The photon index of the latter was also fixed to $1.56\left(\Gamma_{0}\right)$, in the assumption that it is due to reflection from ionised matter of the nuclear radiation. Best fit parameters are listed in Table 1.

In addition, emission lines with Gaussian profiles have been added, as required by the data, from a direct inspection of residuals after any single addition of a spectral component. Any line with a flux not consistent with zero within the error was kept in the model. After each additon an F-test was performed, and we found that all lines are significant at a confidence level of at least $98 \%$, with the only exception of the $1.053 \mathrm{keV}$ line that
Table 1. XMM-Newton and Chandra bestfit parameters (* corresponds to a fixed parameter).

\begin{tabular}{lcc}
\hline \hline Parameter & $\begin{array}{c}X M M-\text { Newton } \\
\text { value }\end{array}$ & $\begin{array}{c}\text { Chandra } \\
\text { value }\end{array}$ \\
\hline$A$ & $1.000^{*}$ & $1.000^{*}$ \\
$A_{\mathrm{Fe}}$ & $1.000^{*}$ & $1.000^{*}$ \\
$N_{\mathrm{H}}\left(10^{21} \mathrm{~cm}^{-2}\right)$ & $4.953_{-0.0019}^{+0.0016}$ & $7.740_{-0.64}^{+0.52}$ \\
$\Gamma_{1}$ & $1.56^{*}$ & $3.7_{-1.4}^{+0.9}$ \\
\hline$\chi^{2} /$ d.o.f. & $302 / 230$ & $294 / 288$ \\
\hline
\end{tabular}

is significant at the $92 \%$ level. Indeed, and similar to Mrk 3 (Bianchi et al. 2005a), the soft X-ray excess is possibly caused by many, unresolved emission lines of the high ionisation states of elements like oxygen, neon, magnesium, and silicon. The addition of many of these lines is in fact suggested by the RGS spectrum, as shown in Fig. 1, even if confusion problems did not allow us to perform a detailed spectral analysis, as said above. To reduce the number of parameters of our model, we fixed the energies of most emission lines at their theoretical values.

All lines have been assumed to be narrow, i.e. with a smaller intrinsic width than the EPIC energy resolution. In practice, we fixed $\sigma=1 \mathrm{eV}$ for all of them, because the source is heavily obscured and lines are supposed to be produced far from the central engine. (We must recall that most of these lines are actually multiplet. For instance, the $\mathrm{Fe} \mathrm{K} \alpha$ line is a doublet, with a separation of about $13 \mathrm{eV}$. However, splitting the line in the fit did not produce any significant improvement of the $\chi^{2}$.) The list of X-ray emission lines with their relative fluxes and their equivalent widths, detected in XMM-Newton spectrum and compared with the Chandra ones (see next section), is given in Table 2. Many of these lines were previously reported by Sambruna et al. (2001b) through Chandra data and confirmed by our new analysis (see next section). In the latter data there is a higher number of lines because of the superior spectral resolution. On the other hand, XMM-Newton data are characterised by a higher $\mathrm{S} / \mathrm{N}$ ratio allowing us to detect lines at lower energies, which are below detectability in Chandra data.

After the inclusion of all lines, the reduced $\chi^{2}$ is 302 for 230 degrees of freedom (d.o.f.). Continuum parameters are kept fixed because we do not find a stable solution of our fit when they are free, while Fig. 2a shows the data with the best fit model and the residuals, and Fig. $2 \mathrm{~b}$ all the spectral components in the model.

\subsection{Chandra data analysis}

The spectral analysis described below refers to the combined spectrum. The spectrum was fitted in the 1-7 keV band, as there is basically no source signal outside this range. The model used to fit Chandra data is the same as the one used for the $X M M-N e w t o n$ analysis. Best fit parameters are listed in Table 1.

The better spectral resolution makes the precise continuum modeling less relevant for identifying emission lines, which is the main goal of our analysis. It was not necessary to fix the line energies to find out a stable solution, as was the case in the previous XMM-Newton analysis. Chandra is less sensitive to energies below about $1 \mathrm{keV}$ than XMM-Newton and consequently it was not necessary to add emission lines of low energies (like oxygen and neon ones) to decribe the soft excess. Chandra X-ray emission lines are summarised in Table 2, together with theoretical energy values, identifications, fluxes, and equivalent widths. All lines have been added as required from eye inspections 
Table 2. Emission lines observed by XMM-Newton EPIC-PN and Chandra with their identification, fluxes, and equivalent widths.

\begin{tabular}{|c|c|c|c|c|c|c|}
\hline $\begin{array}{c}\text { Theoretical } \\
\text { value } \\
\text { keV }\end{array}$ & $\begin{array}{c}\text { Energy } \\
\mathrm{keV}\end{array}$ & $\begin{array}{c}\text { MM-Newton } \\
\text { Flux } \\
{\left[10^{-4} \mathrm{ph} / \mathrm{cm}^{2} / \mathrm{s}\right]}\end{array}$ & $\begin{array}{l}E W \\
\mathrm{eV}\end{array}$ & $\begin{array}{c}\text { Energy } \\
\mathrm{keV}\end{array}$ & $\begin{array}{c}\text { Chandra } \\
\text { Flux } \\
{\left[10^{-4} \mathrm{ph} / \mathrm{cm}^{2} / \mathrm{s}\right]}\end{array}$ & $\begin{array}{l}E W \\
\mathrm{eV}\end{array}$ \\
\hline 0.739 (O VII RRC) & 0.739 & $0.381_{-0.248}^{+0.237}$ & 28.0 & & & \\
\hline 0.826 (Fe XVII L) & 0.826 & $0.416_{-0.141}^{+0.138}$ & 36.4 & & & \\
\hline 0.899 (O VIII RRC) & 0.899 & $0.855_{-0.152}^{+0.141}$ & 85.3 & & & \\
\hline $1.022(\mathrm{Ne} \mathrm{X} \mathrm{K \alpha})$ & 1.022 & $0.249_{-0.010}^{+0.092}$ & 26.9 & & & \\
\hline 1.053 (Fe XXII L) & 1.053 & $0.110_{-0.091}^{+0.080}$ & 11.0 & & & \\
\hline 1.170 (Fe XXIV L) & 1.170 & $0.124_{-0.062}^{+0.0932}$ & 17.0 & & & \\
\hline $1.211(\mathrm{Ne} X \mathrm{~K} \beta)$ & 1.211 & $0.075_{-0.051}^{+0.062}$ & 10.3 & $1.210_{-0.002}^{+0.005}$ & $0.067_{-0.053}^{+0.108}$ & 17.7 \\
\hline $1.331(\mathrm{Mg}$ XI K $\alpha(\mathrm{f}))$ & 1.331 & $0.075_{-0.051}^{+0.0514}$ & 44.0 & $1.332_{-0.002}^{+0.002}$ & $0.101_{-0.067}^{+0.053}$ & 25.2 \\
\hline $1.343(\mathrm{Mg}$ XI K $\alpha(\mathrm{i}))$ & & & & $1.341_{-0.001}^{+0.002}$ & $0.056_{-0.037}^{+0.067}$ & 14.5 \\
\hline $1.352(\mathrm{Mg}$ XIK $\alpha(\mathrm{r}))$ & & & & $1.352_{-0.001}^{+0.001}$ & $0.072_{-0.044}^{+0.071}$ & 19.6 \\
\hline $1.472(\mathrm{Mg}$ XII K $\alpha)$ & & & & $1.472_{-0.001}^{+0.001}$ & $0.081_{-0.047}^{+0.044}$ & 44.2 \\
\hline $1.578(\mathrm{Mg}$ XI K $\beta)$ & & & & $1.579_{-0.001}^{+0.001}$ & $0.034_{-0.016}^{+0.041}$ & 23.8 \\
\hline $1.740+1.745+1.746(\mathrm{Si} I I+\mathrm{Mg}$ XII $+\mathrm{Si}$ VI $)$ & 1.740 & $0.117_{-0.029}^{+0.028}$ & 32.6 & $1.740_{-0.001}^{+0.001}$ & $0.055_{-0.023}^{+0.016}$ & 48.4 \\
\hline $1.839(\mathrm{Si}$ XIII K $\alpha(\mathrm{f}))$ & & & & $1.839_{-0.001}^{+0.001}$ & $0.080_{-0.026}^{+0.0230}$ & 49.2 \\
\hline $1.840(\mathrm{Mg}$ XII $)$ & 1.840 & $0.183_{-0.038}^{+0.051}$ & 49.7 & $1.845_{-0.002}^{+0.001}$ & $0.049_{-0.035}^{+0.026}$ & 27.6 \\
\hline $1.865(\mathrm{Si}$ XIII K $\alpha(\mathrm{r}))$ & 1.865 & $0.082_{-0.051}^{+0.048}$ & 20.5 & $1.864_{-0.001}^{+0.002}$ & $0.077_{-0.029}^{+0.035}$ & 53.0 \\
\hline $2.007(\mathrm{Si}$ XIV K $\alpha)$ & 2.007 & $0.101_{-0.024}^{+0.051}$ & 35.6 & $2.005_{-0.001}^{+0.001}$ & $0.095_{-0.032}^{-0.029}$ & 162 \\
\hline $2.308(\mathrm{Si}$ II) & & & & $2.307_{-0.000}^{+0.001}$ & $0.067_{-0.040}^{+0.032}$ & 105 \\
\hline $2.377+2.387(\mathrm{Si}$ XIV K $\beta+\mathrm{S}$ XII $)$ & 2.377 & $0.191_{-0.029}^{+0.028}$ & 71.4 & $2.394_{-0.024}^{+0.0006}$ & $0.066_{-0.053}^{+0.040}$ & 51.4 \\
\hline $2.430(\mathrm{~S} \mathrm{XV})$ & & & & $2.441_{-0.016}^{+0.024}$ & $0.086_{-0.043}^{+0.053}$ & 95.5 \\
\hline $2.461(\mathrm{Si} \mathrm{XV})$ & 2.461 & $0.119_{-0.026}^{+0.028}$ & 42.3 & $2.462_{-0.006}^{+0.003}$ & $0.085_{-0.040}^{+0.043}$ & 95.6 \\
\hline 2.623 (Si XVI) & 2.623 & $0.069_{-0.023}^{+0.026}$ & 30.9 & & & \\
\hline $2.962(\mathrm{Ar}$ II $)$ & 2.962 & $0.024_{-0.019}^{-0.023}$ & 11.3 & $2.960_{-0.005}^{+0.011}$ & $0.050_{-0.030}^{+0.033}$ & 49.4 \\
\hline 3.104 (Ar XVII) & & & & $3.110_{-0.011}^{+0.005}$ & $0.022_{-0.022}^{+0.030}$ & 21.2 \\
\hline $3.710(\mathrm{Ca}$ II $)$ & 3.710 & $0.054_{-0.019}^{+0.020}$ & 50.0 & $3.690_{-0.000}^{+0.011}$ & $0.038_{-0.018}^{+0.022}$ & 35.8 \\
\hline 6.310 Compton shoulder correction & 6.297 & & & & -0.018 & \\
\hline $6.391+6.405(\mathrm{Fe} \mathrm{K} \alpha)$ & $6.409_{-0.001}^{+0.001}$ & $6.400_{-0.0004}^{+0.0003}$ & 1310 & $6.409_{-0.001}^{+0.001}$ & $2.567_{-0.161}^{+0.131}$ & 1040 \\
\hline $6.637(\mathrm{Fe} X X V)$ & $6.610_{-0.020}^{+0.0014}$ & $0.147_{-0.027}^{+0.029}$ & 172 & & & \\
\hline $6.700(\mathrm{Fe} X X V)$ & $6.658_{-0.048}^{+0.020}$ & $0.108_{-0.026}^{+0.025}$ & 251 & $6.659_{-0.030}^{+0.030}$ & $0.100_{-0.062}^{+0.062}$ & \\
\hline $7.057+7.058(\mathrm{Fe} \mathrm{K} \beta)$ & $7.057^{-0.048}$ & $0.332_{-0.029}^{+0.026}$ & 302 & $7.054_{-0.010}^{+0.0302}$ & $0.332_{-0.096}^{+0.062}$ & 230 \\
\hline $7.461(\mathrm{Ni} \mathrm{K} \alpha)$ & 7.461 & $0.110_{-0.022}^{+0.029}$ & 169 & & & \\
\hline
\end{tabular}
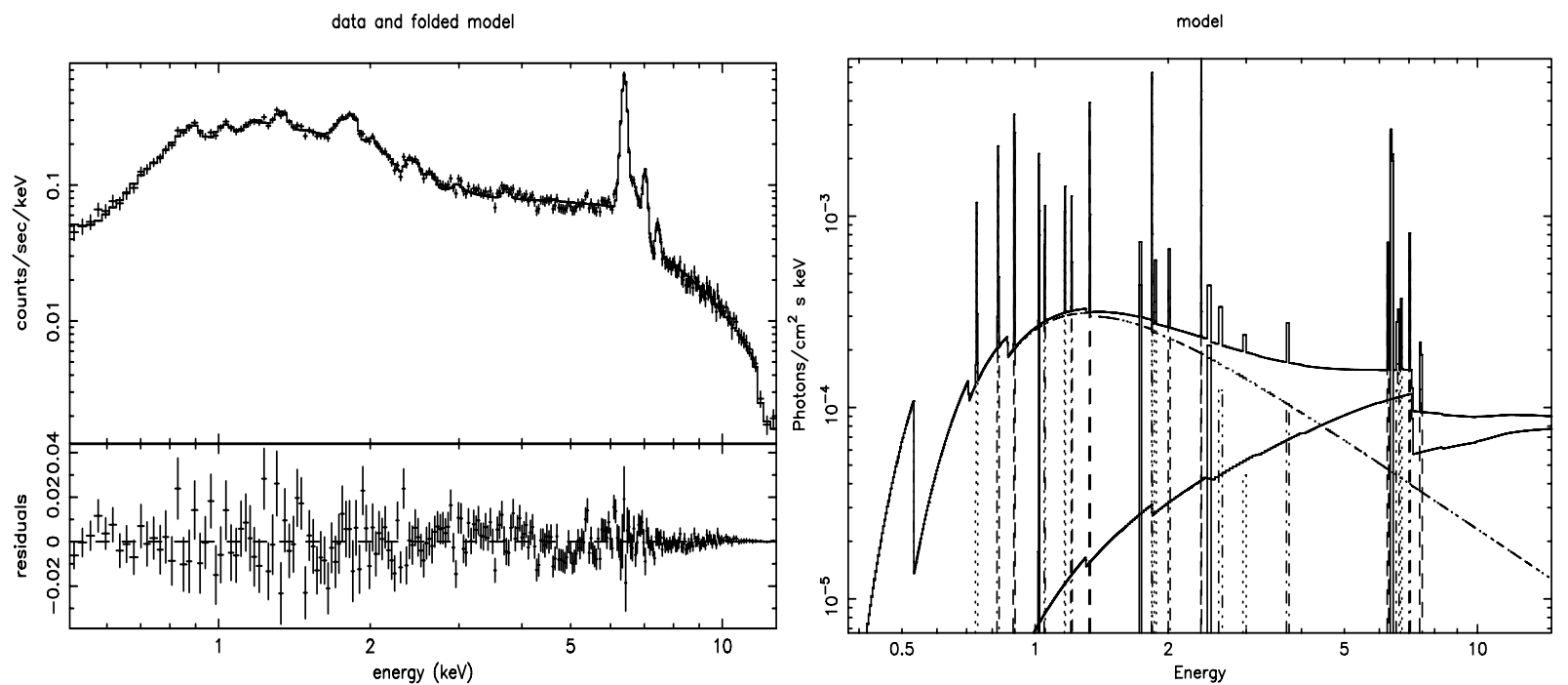

Fig. 2. a) The XMM-Newton EPIC-PN spectrum of the Circinus Galaxy with its residuals, fitted with the bestfit model. b) The XMM-Newton EPIC-PN spectral bestfit model with its components.

of the residuals. As previously done in XMM-Newton analysis, an F-test was performed after each line additon and we found that all lines are significant at a confidence level of at least $99 \%$, with only the exception of the $1.210 \mathrm{keV}$ line significant at the $93 \%$ level. Again they have been assumed narrow, i.e. with $\sigma$ fixed to $1 \mathrm{eV}$. The line spectrum is basically consistent with the $X M M-N e w t o n$ one within the (often large) errors. The major difference is that a few lines detected by XMM-Newton turned out to be a blend, since it was resolved by Chandra. This identification of lines agrees with Sambruna et al. (2001b), obtained with 

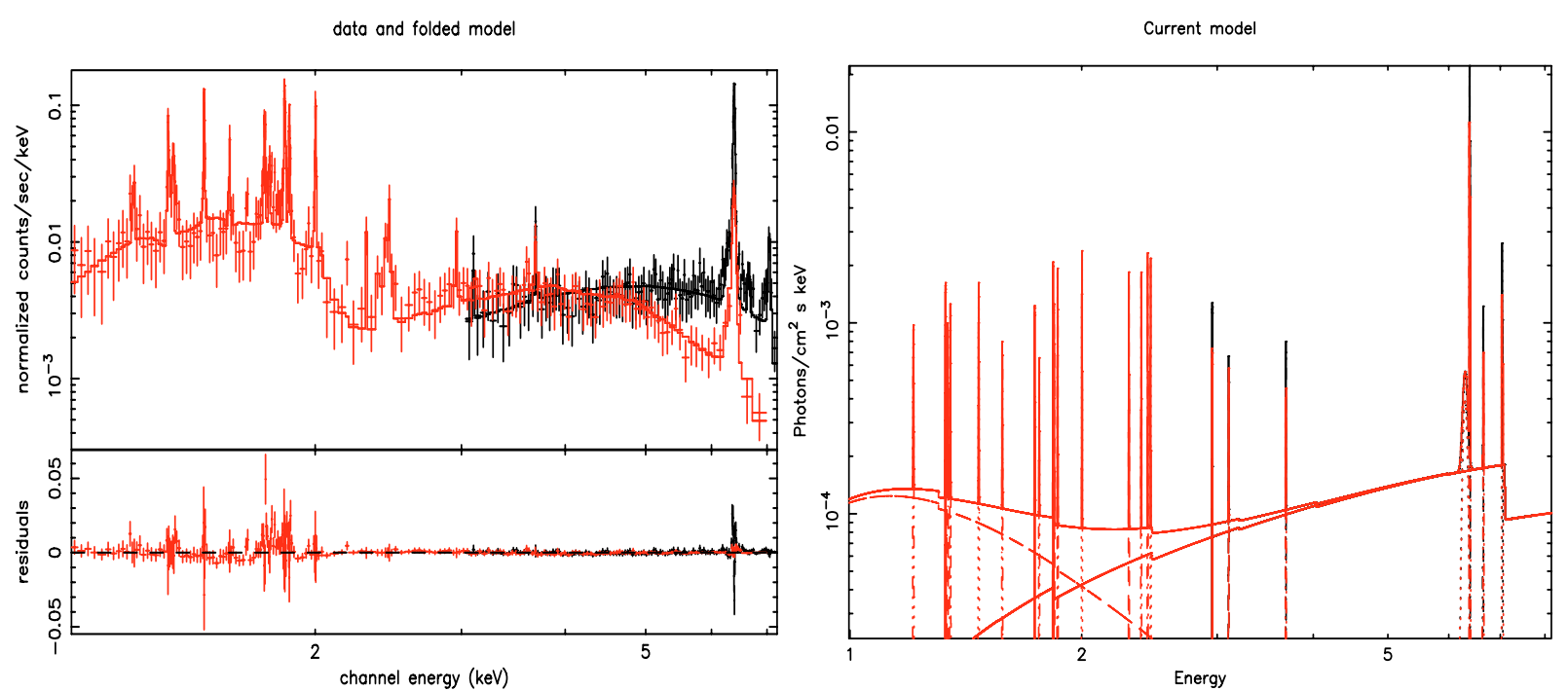

Fig. 3. a) The Chandra MEG (red) and HEG (black) spectrum of the Circinus Galaxy with its residuals, fitted with the best fit model. b) The Chandra spectral best fit model with its components.

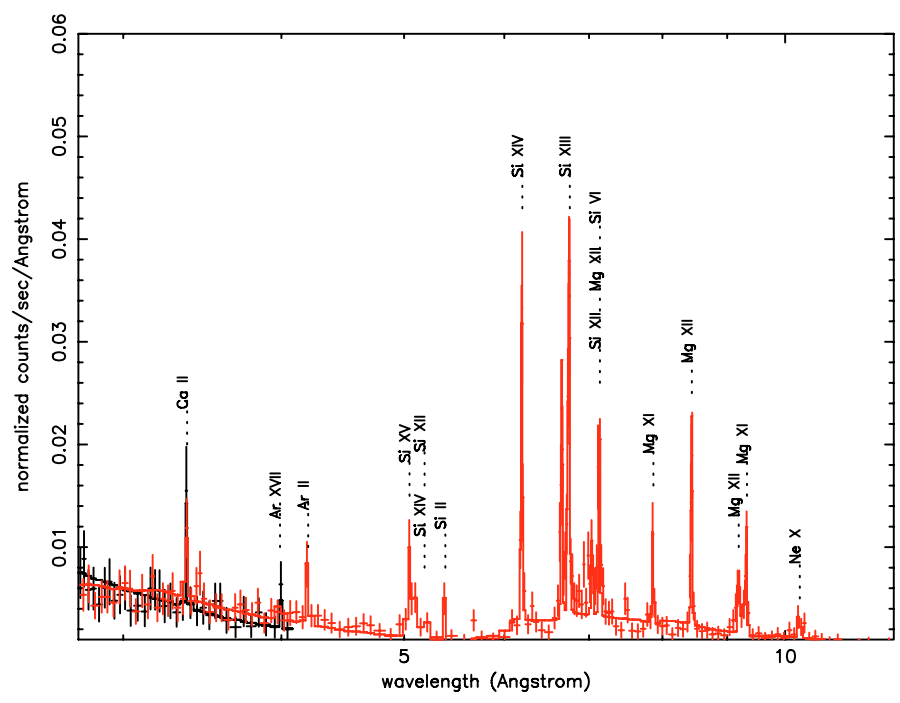

Fig. 4. The soft X Chandra MEG(red) and HEG(black) spectrum.

a subsample of the data we are using here. Also in Chandra data there is a clear presence of the "Compton shoulder" for the iron $\mathrm{K} \alpha$ line, as already found by Bianchi et al. (2003).

Similar to the XMM-Newton analysis, the photon index of the RC (pexrav model) was kept fixed to the values obtained by BeppoSAX observations. After including all lines, the reduced $\chi^{2}$ is 294 for 288 degrees of freedom (d.o.f.). Figure $3 \mathrm{a}$ shows Chandra data with the best fit model and residuals, while Fig. $3 \mathrm{~b}$ shows all the spectral components in the model.

The $N_{\mathrm{H}}$ is $0.774 \times 10^{22} \mathrm{~cm}^{-2}$, so somewhat larger than in the XMM-Newton spectrum. We tried to fit the Chandra data with the same value of the column density of XMM-Newton data, but a worse $\chi^{2}$ value was obtained. In Chandra data the soft excess below $5 \mathrm{keV}$ is also described well by a single power law component, but with a higher spectral index than for XMM-Newton (even if loosely constrained). The reason for this discrepancy is not clear, but it must be recalled that in the XMM-Newton spectrum, especially at low energies, there could still be some contamination from off-nuclear sources, as well as from the $3^{\prime \prime}$ extended emission found by Sambruna et al. (2001a). Of course the much better PSF of Chandra allows such contamination problems to be avoided. Also some differences in the fluxes of low energy emission lines between XMM-Newton and Chandra analysis could be due to the contamination of the off-nuclear sources in the field of view of XMM-Newton.

\section{Photoionisation models}

\section{1. ionisation structure of circumnuclear matter}

We used the well-known numerical code CLOUDY (Ferland 1997) to search for a solution able to reproduce the ionisation states of the circumnuclear matter. The identification of spectral lines, originating in the circumnuclear matter illuminated by the nuclear emission, constrains the ionisation structure of the gas around the nucleus. As far as iron is concerned, the ratio between the iron $\mathrm{K} \alpha$ and $\mathrm{K} \beta$ lines indicates that the ionisation state is below Fe XI (Molendi et al. 2003). For the other elements, constraints on the ionisation state are obtained by the presence of the line of the corresponding ion. For the sake of simplicity in the following analysis, we consider only the most significant elements, as reported in Table 3.

Under the assumption that the reflecting and absorbing material is the same (Bianchi et al. 2001, and references therein), we used CLOUDY to calculate the reflected spectrum from an X-ray illuminated cloud with a slab shape (plane-parallel geometry) with $N_{\mathrm{H}}=4.3 \times 10^{24} \mathrm{~cm}^{-2}$ (Matt et al. 1999). We recall that the dimensionless ionisation parameter

$U=\frac{Q}{4 \pi r^{2} c n}$

where $Q$ is the total number of ionising photons per unit time incident on the slab surface at a distance $r$ from the source; $n$ is the hydrogen number density. With the intrinsic luminosity of Circinus estimated in the $2-10 \mathrm{keV}$ range by Matt et al. (1999), i.e. about $10^{42} \mathrm{erg} \mathrm{s}^{-1}$, we obtained (CGS units):

$U \simeq \frac{1.04 \times 10^{40}}{n r^{2}}$

In our calculation we assumed a value of $16 \mathrm{pc}$ for the distance to the external boundary of the cold slab, $R$, as estimated from 
Table 3. Constraints on the ionisation structure of circumnuclear matter.

\begin{tabular}{ll}
\hline \hline Element & Ranges on the ionisation states \\
\hline Neon & NeX \\
Oxygen & O VII-O IX \\
Magnesium & Mg XI-Mg XII \\
Silicon & Si XII-Si XIV \\
Iron & Fe I-Fe X \\
\hline
\end{tabular}

IR observations (Ruiz et al. 2000) and in agreement with measurements of the dynamical mass (Risaliti et al. 1999), which puts an upper limit on $R$ of about 20 pc. We also fixed the numerical density to $8.7 \times 10^{4} \mathrm{~cm}^{-3}$ on the basis of the further assumption $r \ll R$, found to be justified a posteriori. and of the above given values of the column density and external radius. The inner surface of the slab was found to be:

$r \simeq 0.11 U^{-1 / 2} \mathrm{pc}$.

We searched for a solution with only a single reflecting material, but found no solutions consistent with the constraints in Table 3. In particular, the low ionisation states of high atomic number elements, like iron and nickel, were not compatible with the high ionisation states of lighter elements, like silicon and oxygen. In fact, fluorescent lines should be produced in the first layers of the slab where more abundant ions responsible for the lines should be present. A value of the ionisation parameter low enough to maintain iron in a quasi neutral state cannot ionise silicon (or other light elements) sufficiently to reproduce the ions responsible for the emission lines. Simulations were also performed with the number density declining with radius as a power law with exponents equal to $1.5,2$, and 2.5 , but these models did not produce significantly different results. In Table 4 we summarise the main results of our simulations. The mean ionisation fraction, averaged over a radius corresponding to a Compton depth of about 1 (where the bulk of the observed lines should be produced), is the ratio between the numerical density of a single ion and the total numerical density of the same element. In boldface one can see the most abundant ion for each element and for different ionisation parameters. It is possible to notice that, when $\mathrm{Fe} \mathrm{X}$ is the most abundant iron ion, other lighter elements are not in the ranges imposed by the spectral analysis. These simulations suggested that the spatial structure of the absorbing matter is more complex, as already assumed by Sako et al. (2000) and Sambruna et al. (2001b). In particular we considered the presence of a second reflecting region where emission lines of ionised matter are produced (apart from the Fe XXV line, which requires even more ionised matter), in addition to the dusty torus responsible for $\mathrm{Fe}$ and $\mathrm{Ni}$ lines. This region can be responsible for the low energy powerlaw, which is probably produced by reflection of the nuclear radiation. We point out that the new description of the circumnuclear matter with two reflecting regions, instead of the one with only one reflector, as in Bianchi et al. (2001), is due to the better XMM-Newton and Chandra data compared to those of BeppoSAX and ASCA. In particular, the upper limit to the iron ionisation state is now tighter. This is the main reason the solution with only one reflector is not acceptable. The characteristics of the different reflectors are discussed in the following.

\subsection{Cold reflector: the torus}

By using the maximum value of $U$ for which $\mathrm{Fe} X$ is still the most abundant ion (i.e. $\log U \simeq 0.6$ ), we can derive a lower limit
Table 4. Simulation results: $\log$ of the mean ionisation fraction, averaged on radius, for different elements in various states.

\begin{tabular}{lccccccc}
\hline \hline $\log U$ & Element & VI & VII & VIII & IX & X & XI \\
\hline 0.30 & $\mathrm{Fe}$ & -3.56 & -1.95 & -0.93 & $\mathbf{- 0 . 4 3}$ & -0.49 & -0.83 \\
& $\mathrm{Si}$ & -1.55 & -0.71 & $\mathbf{- 0 . 3 7}$ & -0.54 & -1.19 & -2.25 \\
& $\mathrm{Mg}$ & -1.23 & -0.53 & $\mathbf{- 0 . 3 5}$ & -0.73 & -1.68 & -2.87 \\
& $\mathrm{Ne}$ & $\mathbf{- 0 . 1 8}$ & -0.99 & -1.00 & -1.66 & -3.81 & \\
& $\mathrm{O}$ & -0.46 & $\mathbf{- 0 . 2 2}$ & -1.56 & -3.35 & & \\
\hline 0.40 & $\mathrm{Fe}$ & -3.93 & -2.22 & -1.10 & -0.50 & $\mathbf{- 0 . 4 7}$ & -0.71 \\
& $\mathrm{Si}$ & -1.78 & -0.84 & $\mathbf{- 0 . 3 9}$ & -0.47 & -1.01 & -1.99 \\
& $\mathrm{Mg}$ & -1.42 & -0.62 & $\mathbf{- 0 . 3 4}$ & -0.62 & -1.47 & -2.60 \\
& $\mathrm{Ne}$ & $\mathbf{- 0 . 2 1}$ & -0.92 & -0.84 & -1.41 & -3.46 & -5.85 \\
& $\mathrm{O}$ & -0.53 & $\mathbf{- 0 . 1 9}$ & -1.43 & -3.11 & & \\
\hline 0.43 & $\mathrm{Fe}$ & -4.05 & -2.31 & -1.15 & -0.53 & $\mathbf{- 0 . 4 6}$ & -0.68 \\
& $\mathrm{Si}$ & -1.85 & -0.88 & $\mathbf{- 0 . 4 1}$ & -0.45 & -0.97 & -1.91 \\
& $\mathrm{Mg}$ & -1.48 & -0.65 & $\mathbf{- 0 . 3 4}$ & -0.59 & -1.41 & -2.52 \\
& $\mathrm{Ne}$ & $\mathbf{- 0 . 2 3}$ & -0.90 & -0.80 & -1.33 & -3.36 & -5.72 \\
& $\mathrm{O}$ & -0.55 & $\mathbf{- 0 . 1 8}$ & -1.39 & -3.04 & & \\
\hline 0.50 & $\mathrm{Fe}$ & -4.33 & -2.52 & -1.29 & -0.60 & $\mathbf{- 0 . 4 6}$ & -0.61 \\
& $\mathrm{Si}$ & -2.03 & -0.99 & -0.44 & $\mathbf{- 0 . 4 2}$ & -0.86 & -1.74 \\
& $\mathrm{Mg}$ & -1.63 & -0.73 & $\mathbf{- 0 . 3 5}$ & -0.53 & -1.29 & -2.35 \\
& $\mathrm{Ne}$ & $\mathbf{- 0 . 2 6}$ & -0.87 & -0.71 & -1.17 & -3.13 & -5.42 \\
& $\mathrm{O}$ & -0.60 & $\mathbf{- 0 . 1 6}$ & -1.30 & -2.89 & & \\
\hline 0.60 & $\mathrm{Fe}$ & -4.76 & -2.85 & -1.52 & -0.73 & $\mathbf{- 0 . 4 9}$ & -0.55 \\
& $\mathrm{Si}$ & -2.30 & -1.16 & -0.51 & $\mathbf{- 0 . 3 9}$ & -0.73 & -1.52 \\
& $\mathrm{Mg}$ & -1.86 & -0.86 & $\mathbf{- 0 . 3 8}$ & -0.45 & -1.12 & -2.11 \\
& $\mathrm{Ne}$ & $\mathbf{- 0 . 3 4}$ & -0.85 & -0.59 & -0.97 & -2.83 & -5.01 \\
$\mathrm{O}$ & -0.59 & $\mathbf{- 0 . 1 6}$ & -1.31 & -2.91 & & \\
\hline 0.70 & $\mathrm{Fe}$ & -5.21 & -3.20 & -1.77 & -0.88 & -0.55 & $\mathbf{- 0 . 5 1}$ \\
& $\mathrm{Si}$ & -2.59 & -1.35 & -0.60 & $\mathbf{- 0 . 3 8}$ & -0.63 & -1.31 \\
& $\mathrm{Mg}$ & -2.10 & -1.00 & -0.43 & $\mathbf{- 0 . 4 0}$ & -0.96 & -1.88 \\
& $\mathrm{Ne}$ & $\mathbf{- 0 . 4 5}$ & -0.85 & -0.51 & -0.78 & -2.54 & -4.62 \\
$\mathrm{O}$ & -0.77 & $\mathbf{- 0 . 1 3}$ & -1.07 & -2.46 & & \\
\hline & & & & & & & \\
& & & & &
\end{tabular}

for the inner radius of the dusty torus and obtain a value $\geq$ of 0.05 pc. Bianchi et al. (2001) have found a value (not a lower limit) of about $0.2 \mathrm{pc}$ for the inner radius. Somewhat paradoxically, their looser constraints on the iron ionisation status permitted a tighter constraint on the radius, because they still could find a solution in terms of a single reflector, which in turn implied a relatively narrow range of ionisation parameter to account for the iron and lower $\mathrm{Z}$ elements lines simultaneously. With our better constraints on $\mathrm{Fe}$ ionisation, the single reflector solution is no longer tenable, with the consequence that only an upper limit to the ionisation parameter can be derived.

Our estimate of $r$ is consistent with the value of $0.5 \mathrm{pc}$ estimated from IR observations (Ruiz et al. 2000), and it also agrees with VLT analysis (Prieto et al. 2004). Note also that the assumption $r \ll R$ is, a posteriori, justified.

\subsection{Warm reflector}

Under the hypothesis that the optically thin ionised matter, responsible for the soft X-ray spectrum, can be identified with the [O III] ionisation cone (Greenhill et al. 2003), we can calculate the covering factor of the ionised matter. This hypothesis is supported by recent analysis of 8 nearby Seyfert 2 galaxies observed by HST and Chandra (Bianchi et al. 2006). All sources present soft X-ray emission, due to photoionised gas, which is coincident in extension and overall morphology with the [O III] emission.

We estimated the optical thickness of the warm reflecting region by:

$L_{\text {ref }}=L_{\text {inc }} f \tau$ 
where $L_{\text {ref }}$ is the reflected luminosity $\left(5.9 \times 10^{40} \mathrm{erg} \mathrm{s}^{-1}\right.$ in the $2-10 \mathrm{keV}$ energy range), $L_{\text {inc }}$ the incident luminosity $(1 \times$ $10^{42} \mathrm{erg} \mathrm{s}^{-1}$ in the $2-10 \mathrm{keV}$ energy range) (Matt et al. 1999), $f$ $(\simeq 0.3)$ the covering factor of the ionised matter (estimated from the opening angle of the ionisation cone, i.e. $45^{\circ}$ ), and $\tau$ is its optical thickness. We found $\tau$ a value of 0.2 for the optical thickness, in agreement with the hypothesis of optically thin matter.

\subsection{Hot reflector}

The presence of emission lines of Fe XXV, along with iron lines of high ionisation states around $1 \mathrm{keV}$, cannot be explained by a simple double reflector model. They suggest the presence of a third, "hot" reflector, similar to what is found in NGC 1068 (Iwasawa et al. 1997; Guainazzi et al. 1999; Bianchi et al. 2001). The presence of high ionisation-state lines at low energy has already been observed in other Seyfert 2 galaxies with soft-X excess emission (Bianchi et al. 2006, and references therein). A more detailed discussion of the presence of the emission lines of iron in high ionisation states at about 6.6 and $6.7 \mathrm{keV}$ was reported in the paper of Bianchi et al. (2005b). We can estimate the column density of the hot reflector by using the Montecarlo simulations of Matt et al. (1996), under the assumption of highly ionised material, and a value of $N_{\mathrm{H}}=5 \times 10^{20} \mathrm{~cm}^{-2}$ has been found.

The presence of the hot reflector in the Circinus Galaxy makes it similar to other bright Compton-thick Seyfert $2 \mathrm{~s}$ (Matt et al. 2000), and in particular to NGC 1068 (Ueno et al. 1994; Matt et al. 2004), although the relative intensities of the warm, cold, and hot components are different in the two sources. Spectral features indicating the presence of hot reflecting/absorbing regions have been also observed in some "Compton Thin" sources, as well as in some Seyfert 1 (Bianchi et al. 2005b). To investigate the geometrical properties of these regions, high energy resolution at the iron line energies would be extremely useful. The unfortunate loss of the XRS on board $S u z a k u$ implies that such investigations must wait for several years.

\section{Conclusions}

The XMM-Newton observation of the Seyfert 2 Galaxy Circinus enabled us to describe the properties of its circumnuclear matter.

1. A detailed analysis of the soft X-ray emission has shown the presence of lines from ionised elements of low atomic number that cannot be explained by the same matter producing the fluorescent lines of high atomic number elements ( $\mathrm{Fe} \mathrm{K} \alpha$, $\mathrm{Ni} \mathrm{K} \alpha$ ). The presence of three reflectors is actually required.

2. The first reflector, identifiable as the dusty torus, is able to explain the ionisation structure of iron, as expected by the flux ratio of $\mathrm{Fe} \mathrm{K} \alpha / \mathrm{Fe} \mathrm{K} \beta$ emission lines. The ionisation parameter used to describe the torus structure allowed us to estimate a lower limit for its inner radius of $0.05 \mathrm{pc}$.

3. A second, mildly ionised reflector should be responsible for the line and continuum emission below $5 \mathrm{keV}$. An optical thickness $\tau \simeq 0.2$ can be estimated from the flux of the reflected continuum.
4. Finally, the presence of a third, "hot", highly ionised reflector is required because of the detection of emission lines from highly ionised iron.

Acknowledgements. We thank G. Ferland for his suggestions for the practical use of the numerical code CLOUDY and M. Guainazzi for helpful discussions. Comments from the anonymous referee helped us to make the paper clearer.

\section{References}

Antonucci, R. 1993, ARA\&A, 31, 473

Bianchi, S. 2002, A\&A, 396, 793

Bianchi, S., Matt, G., \& Iwasawa, K. 2001, MNRAS, 322, 669

Bianchi, S., Miniutti, G., Fabian, A. C., \& Iwasawa, K. 2005a, MNRAS, 360, 380

Bianchi, S., Matt, G., Nicastro, F., Porquet, D., \& Dubau, J. 2005b, MNRAS, 357,599

Bianchi, S., Guainazzi, M., \& Chiaberge, M. 2006, A\&A, 448, 499

Brinkmann, W., Siebert, J., \& Boller, T. 1994, A\&A, 281, 355

Brinkmann, A. C., Kaastra, Y. S., van der Meer, R. L. J., et al. 2002, A\&A, 396, 761

Canizares, C. R., Davis, J. E., Dewey, D., et al. 2005, PASP, in press [arXiv:astro-ph/0507035]

Curran, S. J., Johansson, L. E. B., Rydbeck, G., \& Booth, R. S. 1998, A\&A, 338, 863

den Herder, J. W., Brinkman, A. C., Kahn, S. M., et al. 2001, A\&A, 365, L7

Elmouttie, M., Haynes, R. F., Jones, K. L., Sadler, E. M., \& Ehle, M. 1998, MNRAS, 297, 1202

Ferland, G. J. 1997, Internal Report., Univ. Kentucky, Lexington

Freeman, K. C., Karlsson, B., Lynga, G., et al. 1977, A\&A, 55, 445

Greenhill, L. J., Ellingsen, S. P., Norris, R. P., et al. 1997, ApJ, 474, L103

Greenhill, L. J., Booth, R. S., Ellingsen, S. P., et al. 2003, ApJ, 590, 162

Guainazzi, M., Matt, G., Antonelli, L. A., et al. 1999, MNRAS, 310, 10

Iwasawa, K., Fabian, A. C., \& Matt, G. 1997, MNRAS, 289, 443

Jones, K. L., Koribalski, B. S., Elmouttie, M., \& Haynes, R. F. 1999, MNRAS, 302, 649

Krolik, J. H. 1999, Active Galactic Nuclei (Princeton: Princeton Univ. Press)

Krolik, J. H., \& Kallman, T. R. 1987, ApJ, 320, L5

Krolik, J. H., \& Kriss, G. A. 1995, ApJ, 447, 512

Magdziarz, P., \& Zdziarski, A. 1995, MNRAS, 273, 837

Maiolino, R., Krabbe, A., Thatte, N., \& Genzel, R. 1998, ApJ, 493, 650

Marconi, A., Moorwood, A. F. M., Origlia, L., \& Oliva, E. 1994, Messenger, 78, 20

Matt, G. 2002, MNRAS, 337, 147

Matt, G., Brandt, W. N., \& Fabian, A. C. 1996a, MNRAS, 280, 823

Matt, G., Fiore, F., Perola, G. C., et al. 1996b, MNRAS, 208, 253

Matt, G., Fabian, A. C., Guainazzi, M., et al. 2000, MNRAS, 328, 173

Matt, G., Bianchi, S., Guainazzi, M., \& Molendi, S. 2004, A\&A, 414, 155

Molendi, S., Bianchi, S., \& Matt, G. 2003, MNRAS, 343, L1

Moorwood, A. F. M., Lutz, D., \& Oliva, E. 1996, A\&A, 315, L109

Nakai, N., Inoue, M., Miyazawa, K., Miyoski, M., \& Hall, P. 1995, PASJ, 47, 771

Netzer, H. 1996, ApJ, 473, 781

Ogle, P. M., Marshall, H. L., Lee, J. C., \& Canizares, C. R. 2000, ApJ, 545, L81 Oliva, E., Salvati, M., Mooorwood, A. F. M., \& Marconi, A. 1994, A\&A, 288, 457

Oliva, E., Marconi, A., Cimatti, A., \& di Serego Alighieri, S. 1998, A\&A, 329, $\mathrm{L} 21$

Prieto, M. A., Meisenheimer, K., Marco, O., et al. 2004, ApJ, 614, 135P

Risaliti, G., Maiolino, R., \& Salvati, M. 1999, ApJ, 522, 157

Ruiz, M., Alexander, D. M., Young, S., et al. 2000, MNRAS, 316, 49

Sako, M., Kahn, S. M., Paerels, F., \& Liedhal, D. A. 2000, ApJ, 542, 684

Sambruna, R., Brandt, W. N., Chartas, G., et al. 2001a, ApJ, 546, L9

Sambruna, R., Netzer, H., Kaspi, S., et al. 2001b, ApJ, 546, L13

Ueno, S., Mushotzky, R. F., Koyama, K., et al. 1994, PASJ, 46, 3, L71-L75

Veilleux, S., \& Bland-Hawthorn, J. 1997, ApJ, 479, L105 